\title{
Late Term Birth
}

National Cancer Institute

\section{Source}

National Cancer Institute. Late Term Birth. NCI Thesaurus. Code C114096.

Birth at 41 weeks and 0 days through 41 weeks and 6 days. 